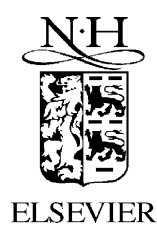

Mathematics and Computers in Simulation 52 (2000) 427-443

MATHEMATICS

AND

COMPUTERS

IN SIMULATION

www.elsevier.nl/locate/matcom

\title{
A general circulation model of the atmosphere using the full-Galerkin method
}

\author{
Jin-Yi Yu \\ Department of Atmospheric Sciences, University of California Los Angeles, 405 Hilgard Avenue, Los Angeles, CA 90095, USA
}

Received 1 April 2000; accepted 1 April 2000

\begin{abstract}
A general circulation model using a full-Galerkin method is developed for the simulation of atmospheric climate and variability. Two variants of the Galerkin method, the spectral-transform method and the finite-element method, are used in this model for the horizontal and vertical representation, respectively. The baroclinic dynamics of this model are examined by performing linear normal mode and nonlinear lifecycle calculations of baroclinic waves. The results suggest that the finite-element method resolves the vertical structure of the baroclinic normal mode better than the finite-difference method. The generation, propagation, and decay of baroclinic waves are well simulated in this model. A long-term integration was carried out with a zonally symmetric forcing applied to the GCM. The simulated climate with a flat topography and that produced in the model hemisphere with an idealized mountain are compared. The results suggest that the presence of mountain does not alter the meridional structure of the zonal mean circulation. Comparisons of these time-mean statistics to observed winter time statistics in the real atmosphere indicate that this GCM produces a reasonable general circulation of the atmosphere. (C) 2000 Published by Elsevier Science B.V. on behalf of IMACS.
\end{abstract}

Keywords: Galerkin method; Atmosphere; General circulation model

\section{Introduction}

In the past few decades, concerns about global climate change and anthropological influences on the environment have encouraged the development of numerical models to simulate and to predict future changes in Earth's climate system. The modeling of the atmosphere is particularly essential because of its active interactions with other components of the climate system, such as oceans and lands. Furthermore, the interactions between atmospheric processes and the hydrological and biochemical cycles have a profound impact on the Earth environment. Numerical models of the atmosphere with various levels of complexity have been developed. Among them, general circulation models (GCMs), which use the primitive equations as the governing equations, offer the most complete description of the dynamics

E-mail address: yu@ atmos.ucla.edu (J.-Y. Yu)

0378-4754/00/\$20.00 (C) 2000 Published by Elsevier Science B.V. on behalf of IMACS.

PII: S 0378-4754(00)00166-X 
of the atmosphere. Therefore, GCMs are considered one of the most powerful tools for modeling the atmospheric climate and its variability.

Several types of numerical techniques have been adopted for the development of atmospheric GCMs. Finite-difference methods were the first to be employed in GCMs (e.g. [1]). With this method, numerical solutions of the governing equations are performed at finite points of grids [14]. The Galerkin method is another way to represent the spatial structures of atmospheric variables and has become more popularly adopted for GCMs. This method uses a limited series of linearly independent basis functions to represent the structures of atmospheric variables, and requires the errors arising from the truncated representations be orthogonal to the basis functions. The unresolved scales in the atmospheric variables are, therefore, not misrepresented in terms of the scales resolved. This Galerkin procedure reduces aliasing problems in numerical approximations and alleviates the nonlinear computational instability.

There are two variants of the Galerkin method: the spectral method and the finite-element method. The spectral method uses non-local continuous functions as the basis functions, and the finite-element method uses piecewise-continuous functions. The use of spectral methods in meteorology date back to Blinova [4] and Silberman [18], but only became popular after the transform technique [5,9] was introduced to compute nonlinear terms. Because of its stability and rational truncation, the spectral-transform method has been more popularly adopted for horizontal representation in atmospheric GCMs. Though finite-difference methods are still widely used for the vertical discretization in atmospheric models, interest in using the finite-element method to represent vertical variations in the atmosphere has been increasing. There are two major reasons for this trend. One reason is that the finite-element method uses locally defined functions as the basis functions, therefore it can fit into any vertical boundary conditions. Another reason is that the finite-element method has higher accuracy than the finite-difference method. When applied on uniform grids, a finite-element approximation produces fourth-order accurate derivatives using only three points [22]. It is third-order accuracy at the most with finite-difference methods. The computational time required for the finite-element method is not much more than the finite difference method.

The advantages of no-aliasing and higher accuracy of the Galerkin methods are particularly favorable for the numerical simulations of atmospheric climate, which requires long-term integrations of atmospheric models. To fully utilize those advantages, a full-Galerkin method GCM is developed in this study. This GCM uses the spectral-transform method for the horizontal representation and the finite-element method for the vertical representation. Since baroclinic dynamics is the most important part of the atmospheric dynamics, a proper modeling of atmospheric baroclinicity is essential for an atmospheric climate model. The normal mode structures and lifecycles of baroclinic waves should be carefully examined to ensure the model has reasonable baroclinic dynamics. In order to focus on the dynamics part of the atmospheric climate, simple parameterizations are used in this model to represent the effects of major physical processes. No moist processes are considered in this study. After the baroclinic dynamics are carefully examined, the model climate are studied with a boundary condition consisting of flat topography and of an idealized mountain.

This paper starts with a brief description of this full-Galerkin method GCM in Section 2. A complete description of this model is given by Yu [24]. The linear baroclinic normal modes simulated by this GCM are studied in Section 3. The nonlinear lifecycle of baroclinic waves produced by this model is examined in Section 4 . The long-term climate produced by this GCM with flat-topography and an idealized mountain are presented in Section 5. Section 6 concludes this study. 


\section{Model description}

This GCM is a global, multi-level, full-Galerkin method model which uses the spectral-transform and finite-element methods for the horizontal and vertical representations, respectively. The primitive equations in the $\sigma$-coordinate of Philips [16] are used as the governing equations, where $\sigma=p / p_{*}$ and $p_{*}$ is the surface pressure. To allow for the use of long time intervals for climate simulations, the semi-implicit time scheme of Robert et al. [17] is adopted. The model has a global domain and multiple levels in the vertical. The horizontal resolution is triangular truncation T21, and there are seven levels in the vertical. These levels are placed at $\sigma=0.10,0.25,0.40,0.55,0.70,0.85$, and 1.00.

The governing equations are the vorticity, divergence, thermodynamic, continuity, and hydrostatic equations

$$
\begin{aligned}
& \frac{\partial \xi}{\partial t}=-\nabla \cdot(\xi+f)+-k \cdot \nabla \times\left(R T \nabla q+\dot{\sigma} \frac{\partial \boldsymbol{V}}{\partial \sigma}-\boldsymbol{F}\right)-v \nabla^{4} \xi \\
& \frac{\partial D}{\partial t}=k \cdot \nabla \times(\xi+f) \boldsymbol{V}-\nabla \cdot\left(R T \nabla q+\dot{\sigma} \frac{\partial \boldsymbol{V}}{\partial \sigma}-\boldsymbol{F}\right)-\nabla^{2}\left(\Phi+\frac{\boldsymbol{V} \cdot \boldsymbol{V}}{2}\right)-v \nabla^{4} D, \\
& \frac{\partial T}{\partial t}=-\nabla \cdot \boldsymbol{V} T+T D+\dot{\sigma} \gamma-\frac{R T}{C_{\mathrm{p}}}\left(D+\frac{\partial \dot{\sigma}}{\partial \sigma}\right)+H_{T}-v \nabla^{4} T, \\
& \frac{\partial q}{\partial t}=-D-\frac{\partial \dot{\sigma}}{\partial \sigma}-\boldsymbol{V} \cdot \nabla q, \\
& \sigma \frac{\partial \Phi}{\partial \sigma}=-R T .
\end{aligned}
$$

The notations used in this paper are listed in Section 7. $v \nabla^{4} \xi, v \nabla^{4} D$, and $v \nabla^{4} T$, are scale selective bi-harmonic diffusion terms, which represent the dissipative effects of the unresolved scales.

The application of spectral-transform method for the horizontal representation of the governing equations and the utilization of semi-implicit schemes in the time differencing are adapted from Daley et al. [7]. Spherical harmonics are used as the basis functions in the spectral representation and have the following forms:

$$
Y_{l}^{m}(\lambda, \mu)=X_{l}^{m}(\mu) \mathrm{e}^{\mathrm{i} m \lambda},
$$

where $X_{l}^{m}$ is the associated Legendre function of the first kind and order $m$. Variables $\xi, D, T, \Phi$ and $q$ are expanded in the triangularly truncated series of spherical harmonics as

$$
A=\sum_{m=-J}^{J} \sum_{l=|m|}^{J} A_{l}^{m} Y_{l}^{m},
$$

where $A_{l}^{m}$ are the spectral coefficients. The spectral representations of the nonlinear terms in Eqs. (1)-(5) are obtained with the transform technique. The variables needed for the calculation of the nonlinear terms are first transformed from the functional space to physical space. The nonlinear products calculated in the physical space are then transformed back to the functional space. The number of Gaussian latitudes has to be $\geq(3 J+1) / 2$, and the number of longitudinal grid points has to be $\geq 3 J+1$. With these numbers 
of grid points, all numerical quadratures are performed exactly with respect to linear and quadratic terms within the retained resolution. The triple products, such as vertical advection terms, are not calculated exactly, but it is believed that aliasing from these terms is not a serious problem for the time integration.

The semi-implicit scheme of Robert et al. [17] is used for the time differencing of the spectral forms of the governing equations. Time derivative terms in the equations are replaced by centered time differences. Those terms that related to the generation and propagation of gravity waves are treated with a backward time scheme to allow for the use of long time steps for climate simulations without causing computational instability. All the other terms are treated with a forward scheme, except the bi-harmonic terms. Forward and trapezoidal schemes are applied to those terms. A weak time filter of Asselin [2] is used to reduce the computational instability that may arise from the leap-frog scheme.

The applications of the finite-element method for the vertical discretization of the governing equations and the boundary conditions in the simple GCM are adopted from Béland and Beaudoin [3]. A one-dimensional Châpeau function is chosen as the basis function. This function has a value of unity at a vertical level and decreases linearly to zero at two neighboring levels. If $e^{i}$ is defined as the basis function at nodal points $\sigma_{i}$ among a set of mesh points $\sigma_{1}, \sigma_{2}, \ldots, \sigma_{N}$, then this basis function can be defined as

$$
e^{i}(\sigma)= \begin{cases}\frac{\sigma-\sigma_{i+1}}{\sigma_{i}-\sigma_{i-1}} & \text { for } \sigma \in\left[\sigma_{i+1}, \sigma_{i}\right], \\ \frac{\sigma_{i+1}-\sigma}{\sigma_{i+1}-\sigma_{i}} & \text { for } \sigma \in\left[\sigma_{i}, \sigma_{i+1}\right] \\ 0 & \text { otherwise. }\end{cases}
$$

The spectral coefficients in the governing equation are expanded in terms of Châpeau functions $e^{k}$ first. For instance, a spectral coefficient $A_{l}^{m}$ can be expanded as

$$
A_{l}^{m}(\sigma) \approx \sum_{k=1}^{L}\left(A_{l}^{m}\right)^{k} \cdot e^{k}(\sigma) .
$$

Here $L$ is the number of sigma levels. The boundary conditions for this $\sigma$ coordinate model require the vertical velocity to vanish at both the top and bottom of the vertical domain, i.e. $\dot{\sigma}=0$ at $\sigma_{1}$ and $\sigma_{N}$.

Three physical processes which are essential to the simulation of atmospheric climate are parameterized in the GCM: the frictional damping due to turbulent processes in the planetary boundary layer (PBL), the adiabatic heating effect of radiative-convective processes, and dry convective adjustment. The damping effect of the PBL is included in the model in the form of nonlinear drags

$$
F_{x}=-C_{\mathrm{D}}|\boldsymbol{V}| \cdot u \text { and } F_{y}=-C_{\mathrm{D}}|\boldsymbol{V}| \cdot v,
$$

Here $|\boldsymbol{V}|$ is wind speed and $C_{\mathrm{D}}$ is the drag coefficient. The value of $C_{\mathrm{D}}$ is maximum at the lowest model layer where it represents the PBL. $C_{\mathrm{D}}$ decreases to trivial values in the interior of the model atmosphere and increases again at the top. The radiative heating effect is included in the model as a Newtonian type of thermal relaxation

$$
H_{T}=\frac{T_{\mathrm{eq}}-T}{\tau} .
$$

Here $T_{\mathrm{eq}}$ is the radiative-convective equilibrium temperature and $\tau$ is the relaxation time. $T_{\mathrm{eq}}$ is given in the form of $\Delta T(\sigma) \times \cos (2 \phi)$, where $\Delta T$ is the equator to pole temperature difference. The vertical 
profile of $\Delta T(\sigma)$ is arranged in such a way that $T_{\text {eq }}$ is statically stable. The third physical process is dry convective adjustment which is used to prevent the model atmosphere from becoming statically unstable. This is done by redistributing the temperature profile with the dry static energy conserved [13].

\section{Linear baroclinic normal modes}

Much understanding on baroclinic dynamics has been obtained through theoretical studies (e.g. $[6,8])$ from the point of view of normal modes of a basic state. This knowledge is used to examine the linear baroclinic dynamics modeled by this full-Galerkin method GCM. Simmons and Hoskins [19] (SH76, hereafter) have conducted such a normal mode calculation using a spectral primitive equation model that employs a finite-difference method for vertical discretization. Their primitive equation model also has a T21 resolution and has eight layers in the vertical. We repeat their normal mode calculation with the full-Galerkin method GCM to assess the impact of finite-element and finite-difference methods of vertical discretization on baroclinic normal modes.

To perform the normal mode calculation, all parameterized physics in the GCM are excluded. The resulting primitive equation model is time integrated from an initial condition which consists of a basic state of $30^{\circ} \mathrm{N}$-jet superimposed with wavenumbers $1-15$ perturbations with very small amplitudes. The $30^{\circ} \mathrm{N}$-jet has the following form:

$$
\bar{u}(\mu, \lambda, \sigma)=\left\{\sin ^{2} \pi \mu\right\} \times U_{0}(\sigma) .
$$

Since the basic state is zonally symmetric, the linearity of the normal mode calculation is maintained by fixing the zonal-mean components in the calculation. The initial temperature and surface pressure fields for this calculation are determined through an initialization process described in [10] to insure computational stability.

Fig. 1 shows the wavenumber dependence of growth rate and phase speed of the normal modes obtained from this GCM. The figure shows that the most unstable mode occurs at wavenumber 8 or 9 . The e-folding time of this mode is about 1.2 days. The phase speed shows little dependence on the wavenumber and is about $6-8^{\circ}$ longitude per day for most of the modes. This wavenumber-independence suggests that basic state advection dominates the zonal propagation of the free baroclinic modes. The wavenumber dependence obtained in this normal mode calculation is similar to that shown in SH76. Differences between the current calculation and that of SH76 appear at the higher wavenumbers. As shown in Fig. 1, the growth rates and phase speeds of these high wavenumber modes are larger in this seven-level model than the eight-layer model of SH76. It is known that higher wavenumber modes have shallower vertical structures and are more sensitive to the vertical resolution. Since vertical discretization is the major difference between our primitive equation model and that of SH76, it is not surprising to see differences appear only in the smaller scale modes.

Fig. 2 displays the vertical structure of the wavenumber 8 mode. The structure is taken from $35.9^{\circ} \mathrm{N}$ where the meridional velocity has its maximum amplitude. This figure shows that the meridional wind has its maximum amplitude at the surface and a secondary maximum in the upper troposphere. For the temperature field, the maximum amplitude also appears at the lowest level, but the secondary maximum in the upper troposphere is not as significant as in the meridional wind field. The largest vertical (omega) velocity occurs in the middle of the model troposphere. The near-surface maxima shown in these normal mode structures are consistent with the linear theory of Charney [6], which showed that the normal mode 

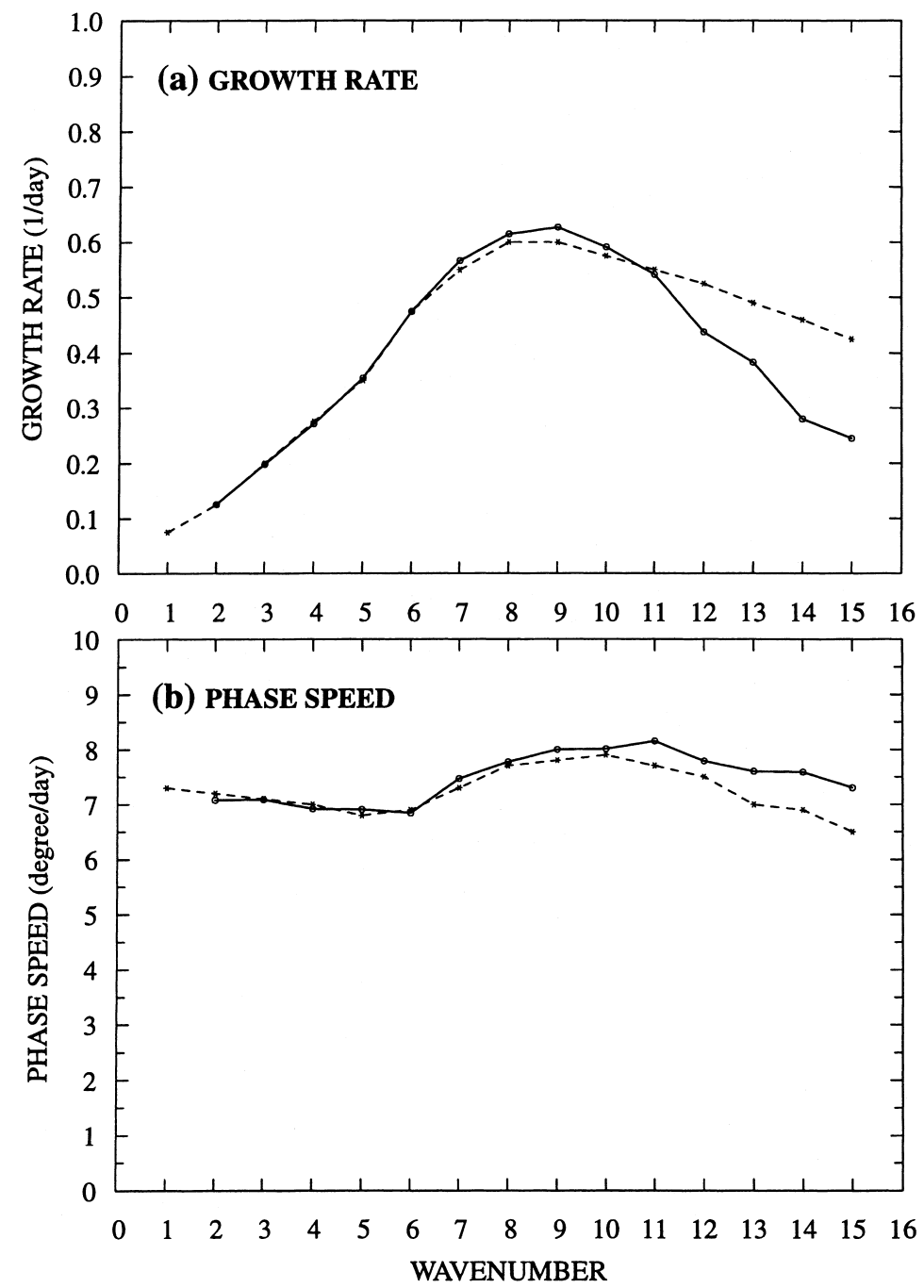

Fig. 1. Wavenumber dependence of (a) growth rate and (b) phase speed of the normal modes. The solid curves are results from the normal mode calculation by the full-Galerkin method GCM, and the short-dash curves are results from similar calculation in a primitive equation model of Simmons and Hoskins [19]. In the latter calculation, T21 and eight-layer resolutions are used.

has the maximum meridional wind at the surface. The vertical structure shown here is also similar to those obtained by SH76, except that they did not produce the secondary upper-tropospheric maxima in meridional wind and temperature.

Simmons and Hoskins [20] seek to better resolve normal mode structures in the upper troposphere by increasing the vertical resolution of the primitive equation model of SH76. When the vertical resolution was increased to 13 vertical layers, they obtained a larger amplitude of the meridional wind maximum there. The normal mode obtained from our finite-element method primitive equation model is closer to the higher-resolution results of Simmons and Hoskins [20] than to SH76 (see Fig. 2). Since the vertical 

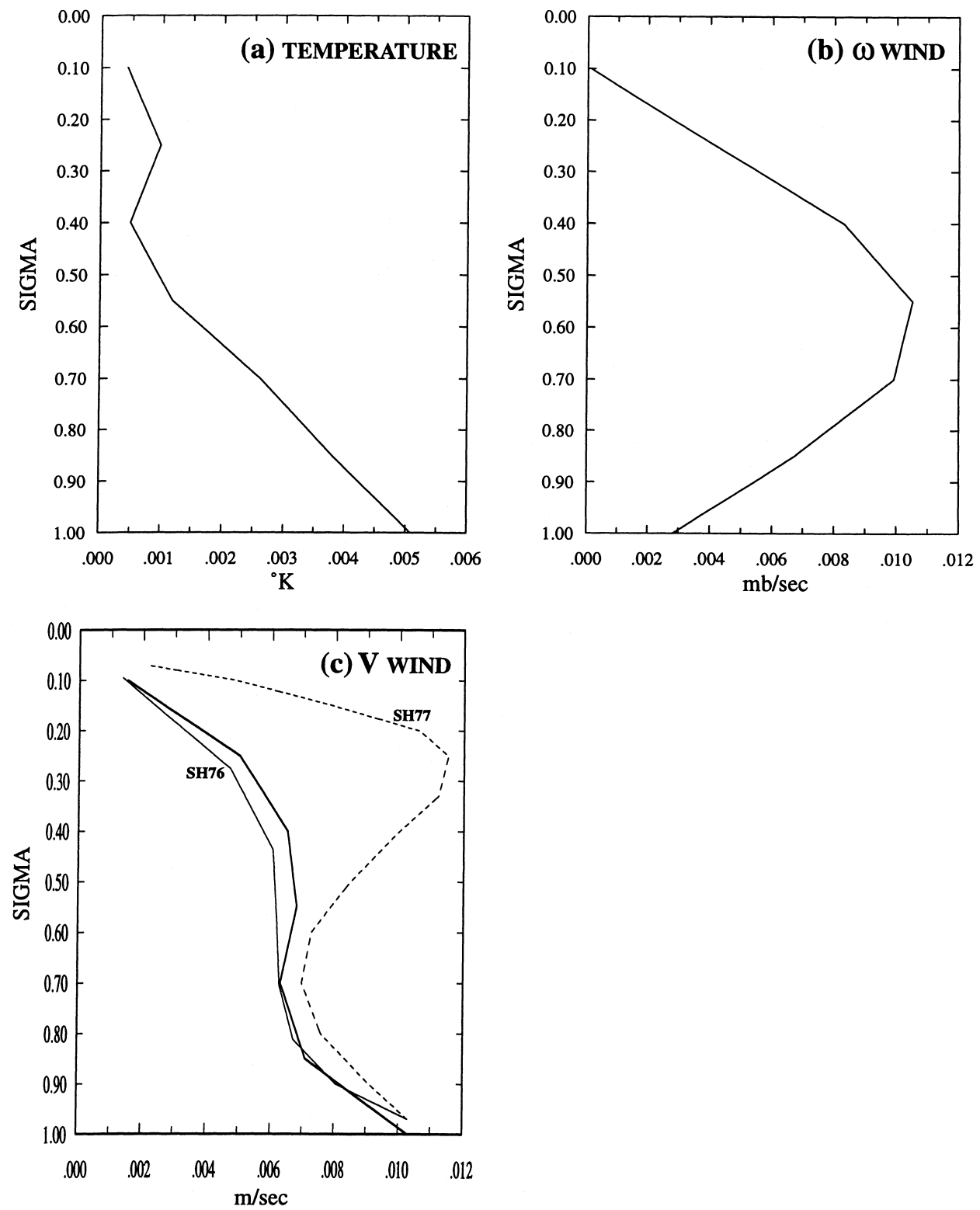

Fig. 2. Vertical structures of the amplitude of (a) temperature, (b) omega velocity, and (c) meridional wind for wavenumber 8 normal mode. Values in panel (a), (b), and the thick solid curve in panel (c) are plotted at $35.9^{\circ} \mathrm{N}$ where the meridional velocity has its maximum amplitude. The thin curves in panel (c) are the vertical structures of meridional wind taken from Simmons and Hoskins [19] (Fig. 9, the solid curve) and from Simmons and Hoskins [20] (Fig. 3, the dashed curve).

resolutions used in SH76 and in our calculation are similar, the finite-element method used in this GCM apparently resolves the vertical structures better than the finite-difference method used in SH76. The higher accuracy of the finite-element method in representing the vertical structures is likely to be the cause. 


\section{Nonlinear lifecycle of baroclinic waves}

The linear baroclinic modes obtained from normal mode calculation have their maximum amplitudes near the surface. This is inconsistent with observed baroclinic waves whose amplitudes are maximum in the upper troposphere. Increasing the vertical resolution, as has been done by Simmons and Hoskins [20], does not resolve this discrepancy. The differences between the normal modes and observed waves suggest that the behavior of finite-amplitude baroclinic waves cannot be described completely by the linear normal modes. Simmons and Hoskins [21] (SH78, hereafter) studied the temporal evolution of finite-amplitude baroclinic waves with the same primitive equation model of SH76. They noticed that baroclinic waves experience a lifecycle of growth and decay. They also found that the lifecycle averaged properties of baroclinic waves are in better agreement with the observations than the linear normal mode structures. In addition, the interactions between the zonal mean flow and finite-amplitude baroclinic waves play an important role in the atmospheric general circulation. It is necessary to make sure that a climate model simulates the nonlinear behavior of baroclinic waves in a realistic manner. The nonlinear baroclinic waves simulated by this GCM are examined with a lifecycle calculation presented in this section. The initial condition for this calculation consists of the same $30^{\circ} \mathrm{N}$-jet basic state and a small amplitude wavenumber 6 perturbation. The perturbation has an amplitude of $1 \times 10^{-6} \mathrm{~s}^{-1}$ in relative vorticity. Bi-harmonic diffusion terms are included in this lifecycle calculation to dissipate enstrophy accumulating at the truncated scales due to the occlusion of baroclinic waves. The e-folding time of this diffusion is 0.75 days on total wavenumber 21 . The basic state is allowed to change in this lifecycle calculation.

The $30^{\circ} \mathrm{N}$-jet basic state with initial wavenumber 6 perturbations is integrated for 50 days with this full-Galerkin method GCM. The evolution of eddy kinetic energy (EKE) is used as a basis for defining the stages of the lifecycles of the wavenumber 6 wave. This quantity is calculated using the formula

$$
\mathrm{EKE}=\int_{\sigma_{1}}^{1}\left\{\int_{0}^{1}\left(\int_{0}^{2 \pi} \frac{1}{2}\left(U^{* 2}+V^{* 2}\right) a \cos \phi \mathrm{d} \lambda\right) a \mathrm{~d} \mu\right\} \frac{p_{\mathrm{s}}}{g} \mathrm{~d} \sigma,
$$

where $p_{\mathrm{s}}$ is the surface pressure. It is a hemispherically averaged and vertically weighted quantity. Fig. 3a shows the time evolution of EKE from the lifecycle calculation shown, along with the variations of the maximum eddy momentum flux and the maximum eddy heat flux. A well defined lifecycle, characterized by a growing phase between days 0 and 10 and a decaying phase afterward, is evident. In the growing phase, the eddy heat flux is the dominant process and reaches its maximum values 1 day before the baroclinic wave is mature. After the wave kinetic energy reaches a maximum, the eddy momentum flux becomes the dominant term in the energetics. This figure suggests a lifecycle consisting of baroclinic growth and barotropic decay.

The energy cycle of Lorenz [12] is used to show how baroclinic or barotropic processes relate to variations in eddy kinetic energy in the lifecycle calculation. In Lorenz's energy cycle, two conversion terms determine the change of eddy energy: the energy conversion between eddy available energy and eddy kinetic energy $\left(C_{\mathrm{E}}\right)$ and the conversion between eddy kinetic energy and zonal kinetic energy $\left(C_{\mathrm{K}}\right)$. These two conversion terms can be calculated as

$$
C_{\mathrm{E}}=-\frac{R}{g} \int_{P_{2}}^{1000}\left(\int_{S} \omega^{*} T^{*} \mathrm{~d} S\right) \frac{\mathrm{d} p}{p}
$$




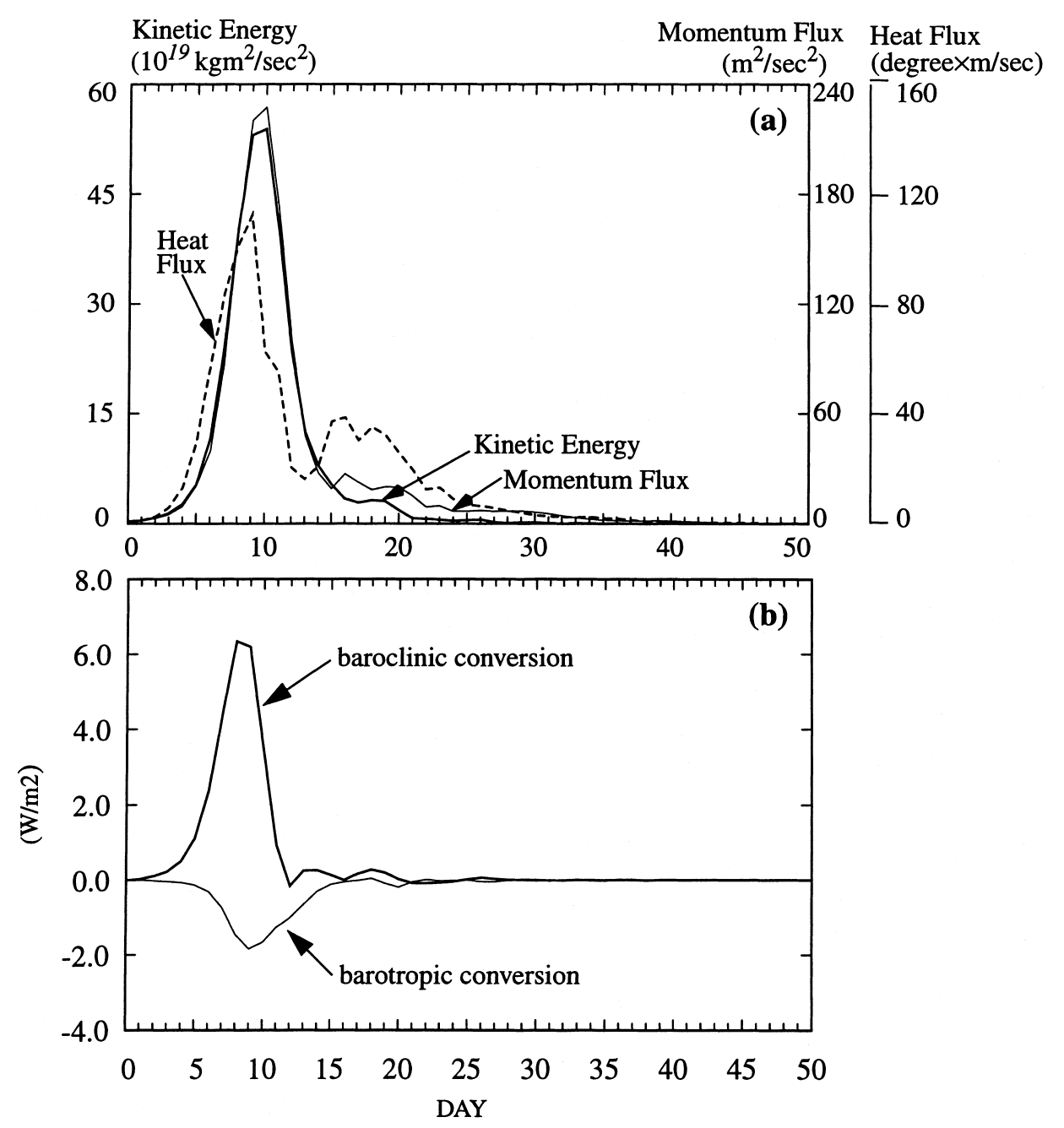

Fig. 3. Temporal evolution of (a) hemisphere-integrated kinetic energy, the maximum momentum flux, and the maximum heat flux and (b) baroclinic and barotropic energy conversions for wavenumber 6 perturbation from the lifecycle calculation. In panel (a), the thick solid curve represents the eddy kinetic energy, the short-dash curve the maximum eddy heat flux and the thin solid curve the maximum eddy momentum flux. In panel (b), the thick solid line represent the baroclinic energy conversion, and the thin solid line represents the barotropic energy conversion.

and

$$
C_{\mathrm{K}}=-\frac{1}{g} \int_{P_{2}}^{1000}\left(\int_{S} \cos \phi\left\{\overline{\frac{u^{*} v^{*}}{a}} \frac{\partial}{\partial \phi}\left(\frac{\bar{u}}{\cos \phi}\right)+\overline{u^{*} w^{*}} \frac{\partial}{\partial p}\left(\frac{\bar{u}}{\cos \phi}\right)\right\} \mathrm{d} S\right) \mathrm{d} p .
$$

Here the overbar represents a zonal mean, ()$^{*}$ is a eddy quantity, and $\int_{S}$ is a surface average over a hemisphere. The temporal variations of these two terms are shown in Fig. 3b. The conversion from eddy available energy to eddy kinetic energy dominates the growing phase, and the baroclinic wave decays by converting eddy kinetic energy to zonal kinetic energy. The barotropic energy conversion 


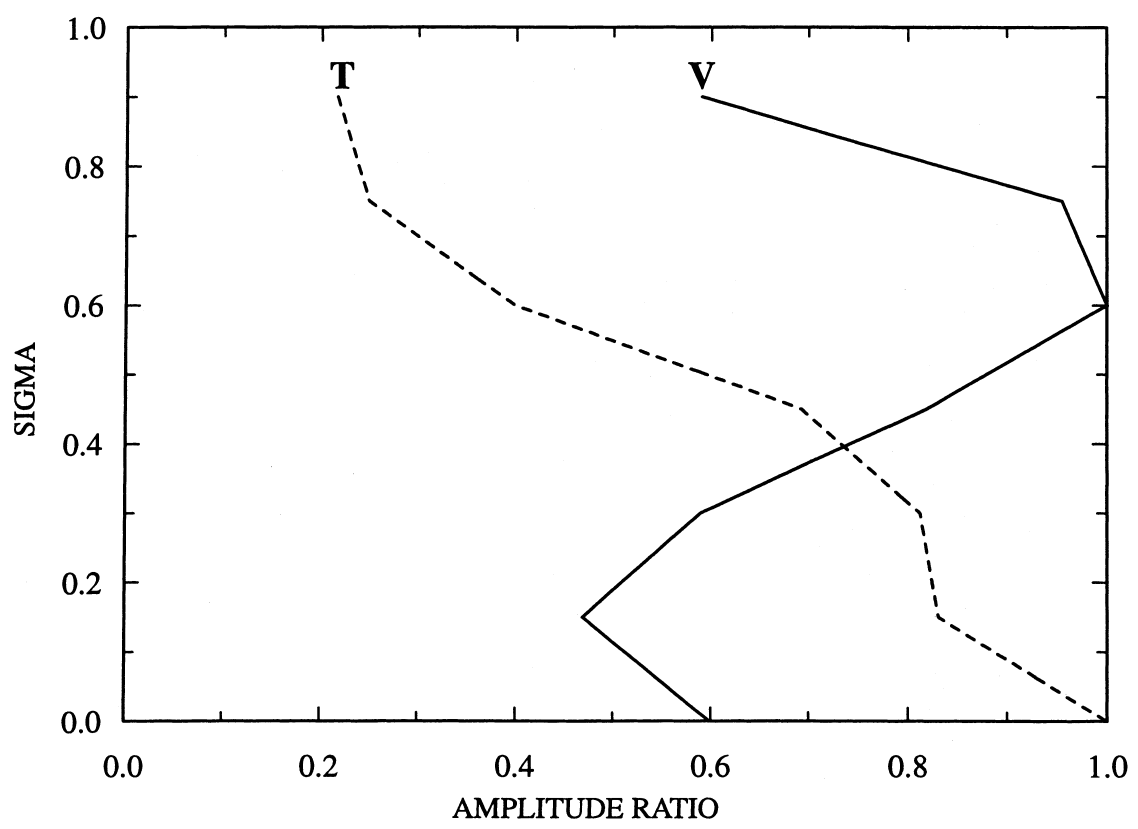

Fig. 4. Vertical structure of the lifecycle-averaged wavenumber 6 perturbation in the lifecycle calculation. The values displayed are the ratios of the maximum amplitude of each vertical level to the largest value in the vertical. The solid curve represents the amplitude ratio of meridional wind and, the short-dash curve represents amplitude ratio of temperature.

rate is only one-third that of the baroclinic energy conversion. From Fig. $3 a$ and $b$, it is clear that the lifecycle of the wavenumber 6 perturbation is characterized by baroclinic growth and barotropic decay.

The lifecycle averaged vertical structure for wavenumber 6 is shown in Fig. 4. The average is taken from days 5 to 15 . It includes 5 days from the growing phase and 5 days from the decaying phase. The maximum meridional winds occurs in the upper troposphere, rather than near the surface as is the case for the normal mode. There is a secondary maximum at the surface, however, although it is much weaker than the upper-level maximum. The temperature field still has its largest amplitude near the surface, but it decreases in the vertical much more slowly than in the linear case. Compared to the structure in the linear calculation, it is seen that the vertical structure of the nonlinear baroclinic wave is obviously closer to that of observed baroclinic waves.

The variation of the horizontal wave structure during the lifecycle calculation is examined in Fig. 5. This figure displays the upper tropospheric streamfunction for the wavenumber 6 perturbation during its growing, mature, and decaying phases. In the growing phase, the wave exhibits a southwest to northeast tilt equatorward of the $30^{\circ} \mathrm{N}$-jet and an opposite tilt on the poleward side of the jet. When the eddy kinetic energy reaches its maximum on day 10, the northwest-southeast tilt of the bean-shaped structure weakens. The weakening of the tilt to the north of the jet continues in the decaying phase. On day 12, the baroclinic wave assumes a southwest to northeast tilt at most latitudes. Also seen in the decaying phase is an equatorward shift of about $10^{\circ}$ of the streamfunction maxima. This suggests equatorward propagation of the baroclinic wave as it decays. 


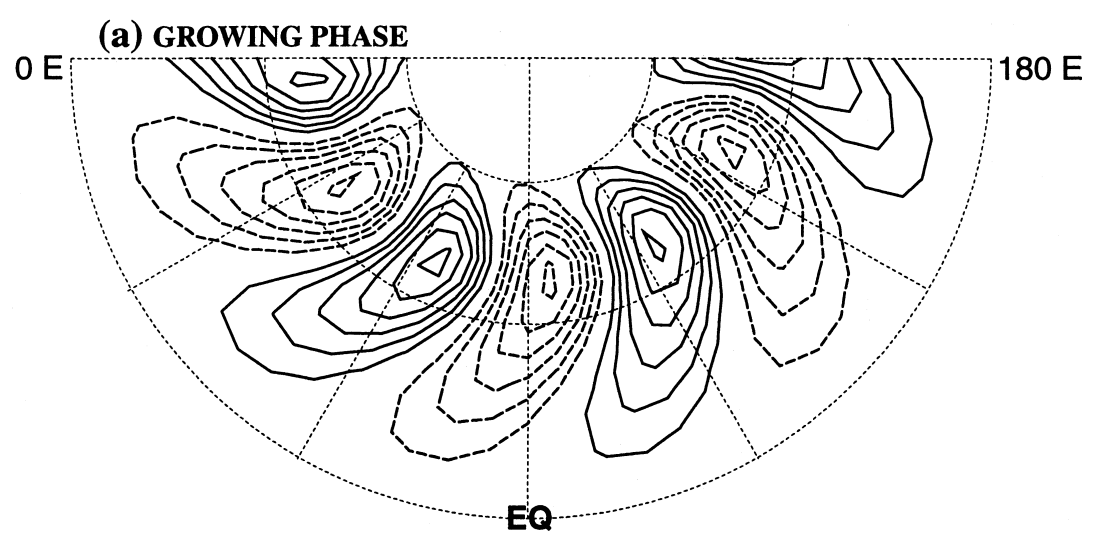

(b) MATURE PHASE

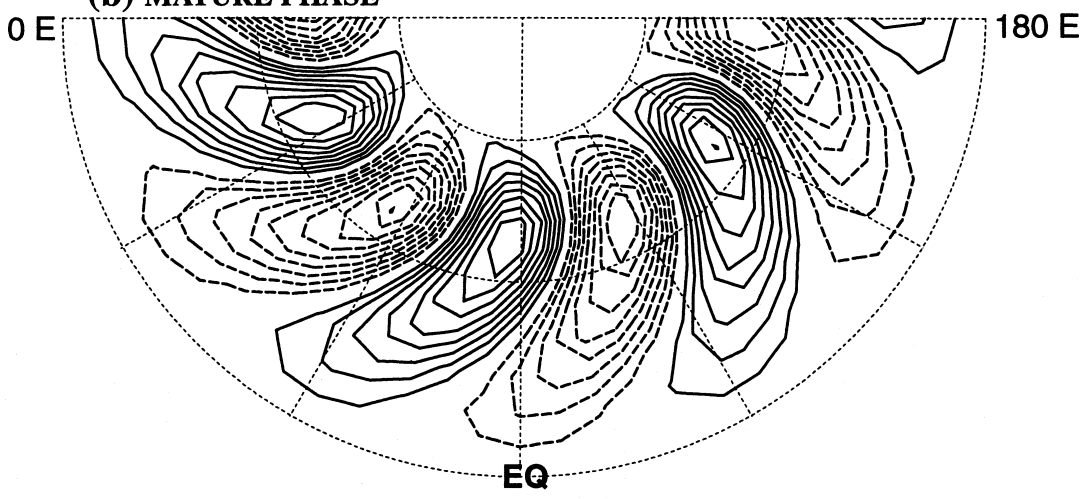

(c) DECAYING PHASE

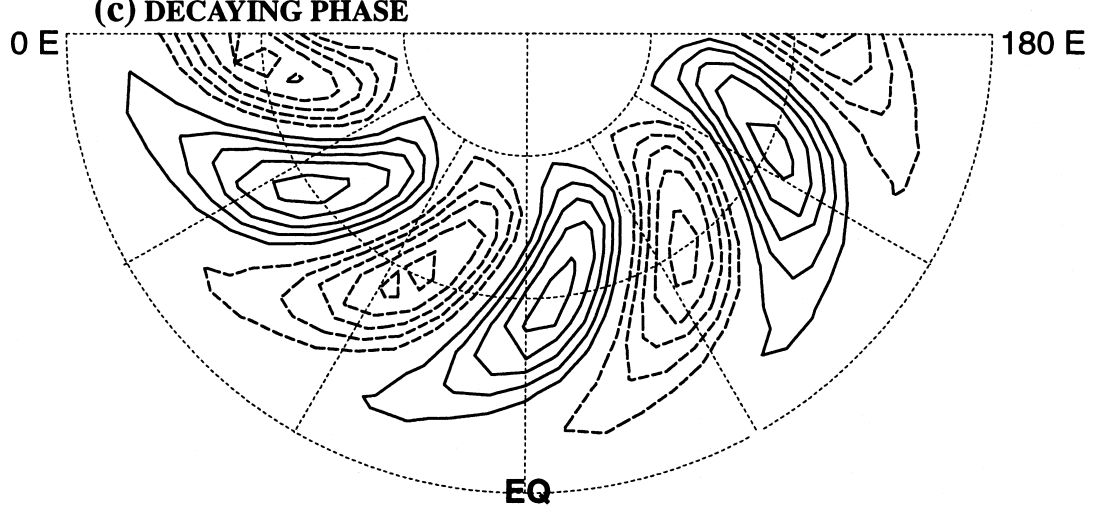

Fig. 5. Horizontal structure of streamfunction for wavenumber 6 perturbation in the (a) growing phase (day 7), (b) mature phase (day 10), and (c) decaying phase (day 12) of its lifecycle. The structure is plotted at $\sigma=0.25$. Contour intervals are $2 \times 10^{6} \mathrm{~m}^{2} / \mathrm{s}$ in all three panels.

\section{Climate in no-mountain and mountain hemispheres}

The climate of this GCM is examined with an long-term integration. To gain insight into the effect of large-scale mountains on the atmospheric circulation, a Gaussian-shaped mountain is placed in the 


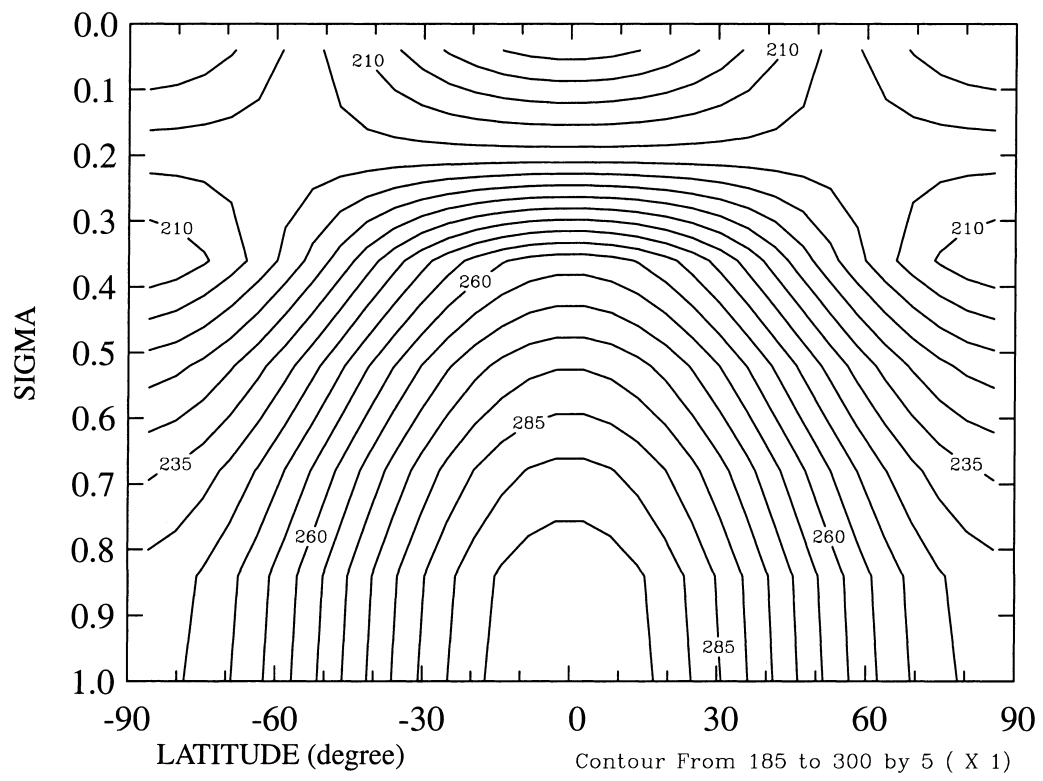

Fig. 6. Meridional structure of the equilibrium temperatures used in the thermal relaxation for the mountain/no-mountain sumulation. The contour interval is $5^{\circ} \mathrm{K}$.

northern model hemisphere. The southern model hemisphere has no topography. The Gaussian-shaped mountain is prescribed as

$$
Z(\sigma)=Z_{0} \exp \left(-\left\{\left(\frac{\lambda-\lambda_{0}}{\Delta \lambda}\right)^{2}+\left(\frac{\phi-\phi_{0}}{\Delta \phi}\right)^{2}\right\}\right) .
$$

Here $Z_{0}$ is the height of the mountain; $\left(\lambda_{0}, \phi_{0}\right)$ are the centered longitude and latitude of the mountain; and $(\Delta \lambda, \Delta \phi)$ are the widths of the mountain in longitude and in latitude. In this simulation the mountain height is $Z_{0}=4 \mathrm{~km},\left(\lambda_{0}, \phi_{0}\right)=\left(180^{\circ} \mathrm{E}, 40^{\circ} \mathrm{N}\right)$, and $(\Delta \lambda, \Delta \phi)=\left(30^{\circ}, 15^{\circ}\right)$. The height and location of the mountain are chosen to represent the Himalayas. The integration is started from a rest, and isothermal atmosphere. A zonally symmetric forcing in the form of thermal relaxation is applied. The equilibrium temperatures to which the model atmosphere is relaxed are shown in Fig. 6. These temperatures have a $\cos (2 \phi)$ profile in latitude, with equator-to-pole temperature differences of $60^{\circ}$ at most model levels. These idealized equilibrium temperatures are used to resemble the winter-time atmosphere. The model is spun up for 400 days from its initial state, and the end of this integration is used as the initial condition for another 3070-day integration. Time mean statistics are obtained by averaging the whole 3070 days of this integration.

The meridional structure of time averaged zonal-mean zonal winds from the integration is shown in Fig. 7. The distributions of zonal wind in both the no-mountain hemisphere and the mountain hemisphere are quite similar. The jets in the model are located at $40^{\circ}$ in both hemispheres, with maximum wind speeds around $45 \mathrm{~m} / \mathrm{s}$. The presence of a large-scale mountain in the northern model hemisphere does not affect the location and the maximum wind speeds of zonal-mean jets very much. The 10-year (1963-1973) averaged statistics from Oort [15] show that the jets in the Northern Winter Hemisphere and in the Southern Winter 


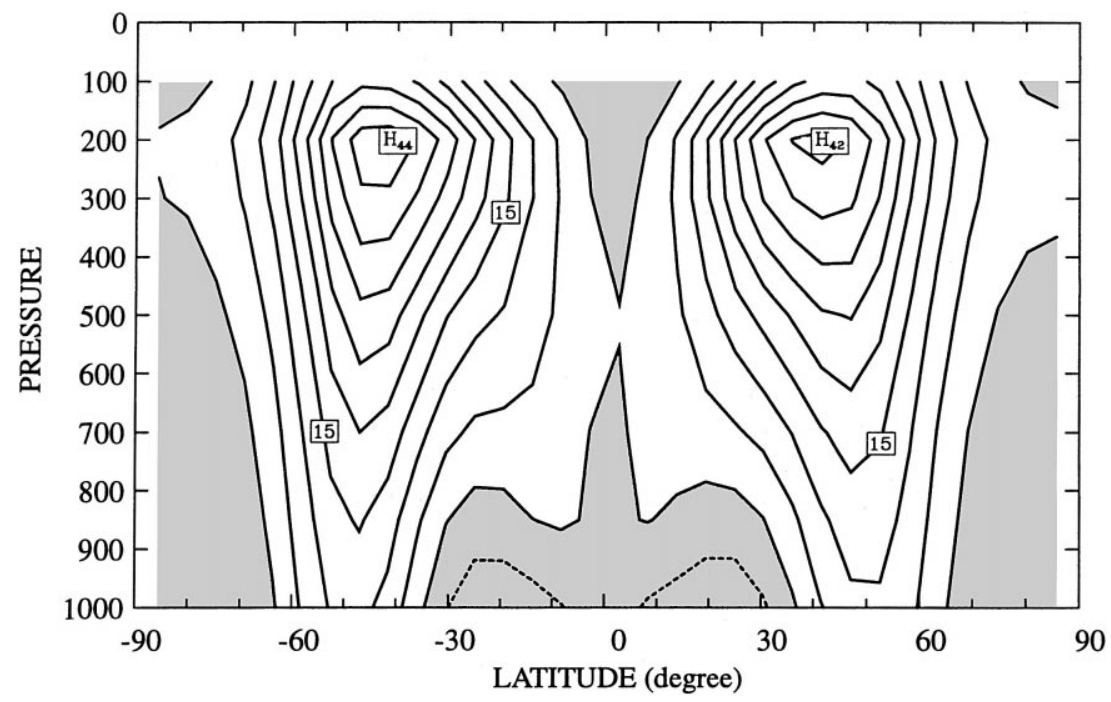

Fig. 7. Meridional structure of time-mean Zonally averaged zonal winds from the 3070-day integrations. Contour interval is $5 \mathrm{~m} / \mathrm{s}$, and easterlies are shaded.

Hemisphere are both located at $30^{\circ}$. Similar to our GCM simulation, the presence of the Himalayas and the Rockies in the Northern Hemisphere do not alter the location of the zonal-mean jet from that in the Southern Hemisphere. Though the presence of large-scale orography does not affect upper level jets, it is clear from Fig. 7 that the magnitude of surface westerlies is affected by the topography. The maximum surface westerlies in the mountain hemisphere are less than $10 \mathrm{~m} / \mathrm{s}$, but are more than $10 \mathrm{~m} / \mathrm{s}$ in the no-mountain hemisphere. The weaker surface winds in the model's mountain hemisphere is in agreement with observations. Surface winds in the Southern Hemisphere are stronger than in the Northern hemisphere during the same seasons. Wallace [23] argued that this difference is a result of the additional mountain torque in the Northern Hemisphere. Though the mean zonally averaged zonal winds show very few differences between the mountain and in the no-mountain hemispheres, their two-dimensional horizontal structures exhibit significant influences of the underlying orography. Fig. 8 shows the time-mean zonal wind at $300 \mathrm{mb}$. As expected, a zonally symmetric jet is observed in the no-mountain hemisphere while a strong localized jet appears in the mountain hemisphere. The localized jet in the mountain hemisphere is about $60^{\circ}$ downstream from the Gaussian-shaped mountain. Equatorward of this westerly jet, there is a localized easterly jet. According to linear theory, stationary Rossby wave trains excited by orography and propagate northward and southward away from the mountain. Localized westerly and easterly jets are formed downstream from the forcing. The longitudinal locations of the midlatitude jet and the tropical jet in the model are basically consistent with this linear theory of stationary waves.

The meridional structure of the time-mean meridional wind field (Fig. 9) in the mountain and in the no-mountain hemispheres are similar, with a distinct three cell structure. The lower branches of the cells are confined in a shallow layer between the surface and $900 \mathrm{mb}$. The presence of a large-scale mountain in the northern hemisphere does not change the distribution and strength of meridional winds. But the horizontal distributions of meridional wind are very different between the two hemispheres of the GCM (Fig. 10). A strongly zonally asymmetric distribution is seen in the mountain hemisphere, especially near 


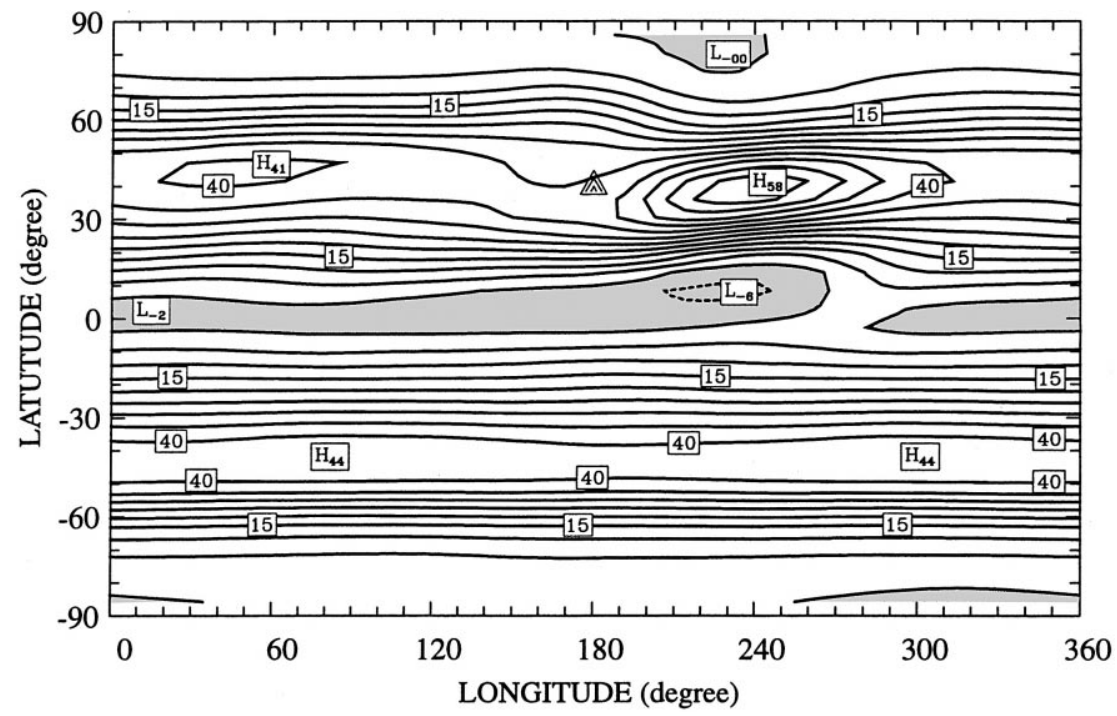

Fig. 8. Time-mean 300 mbar zonal wind from the 3070-day integration. Contour interval is $5 \mathrm{~m} / \mathrm{s}$, and easterlies are shaded. The center of the mountain is indicated by a triangular symbol.

and downstream of the mountain. Strong surface southerly and northerly winds are located immediately upstream and downstream of the mountain, respectively. In primitive equation models, topographic effects are imposed on the model atmosphere through the boundary condition in the thermodynamic equation. As discussed by Hoskins and Karoly [11], the adiabatic cooling due to the rising of the air upstream of

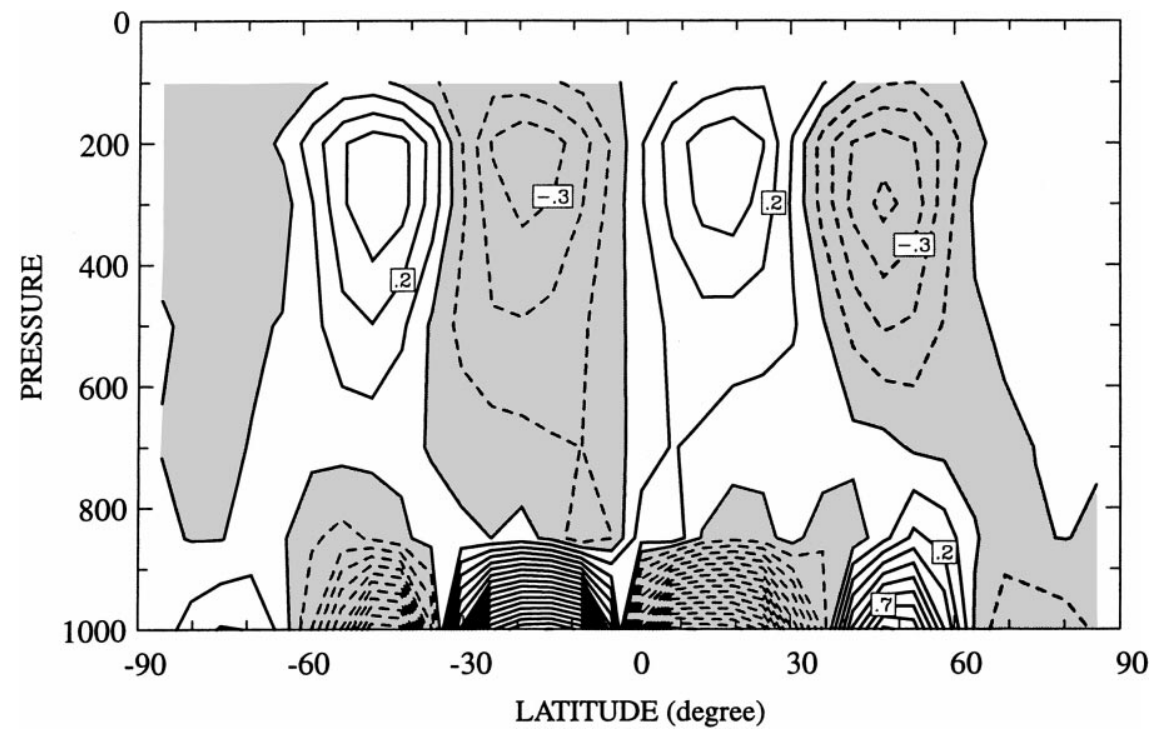

Fig. 9. Vertical cross section of the time-mean zonally averaged meridional winds from the 3070-day integration. Contour interval is $1 \mathrm{~m} / \mathrm{s}$, and southerlies winds are shaded. 


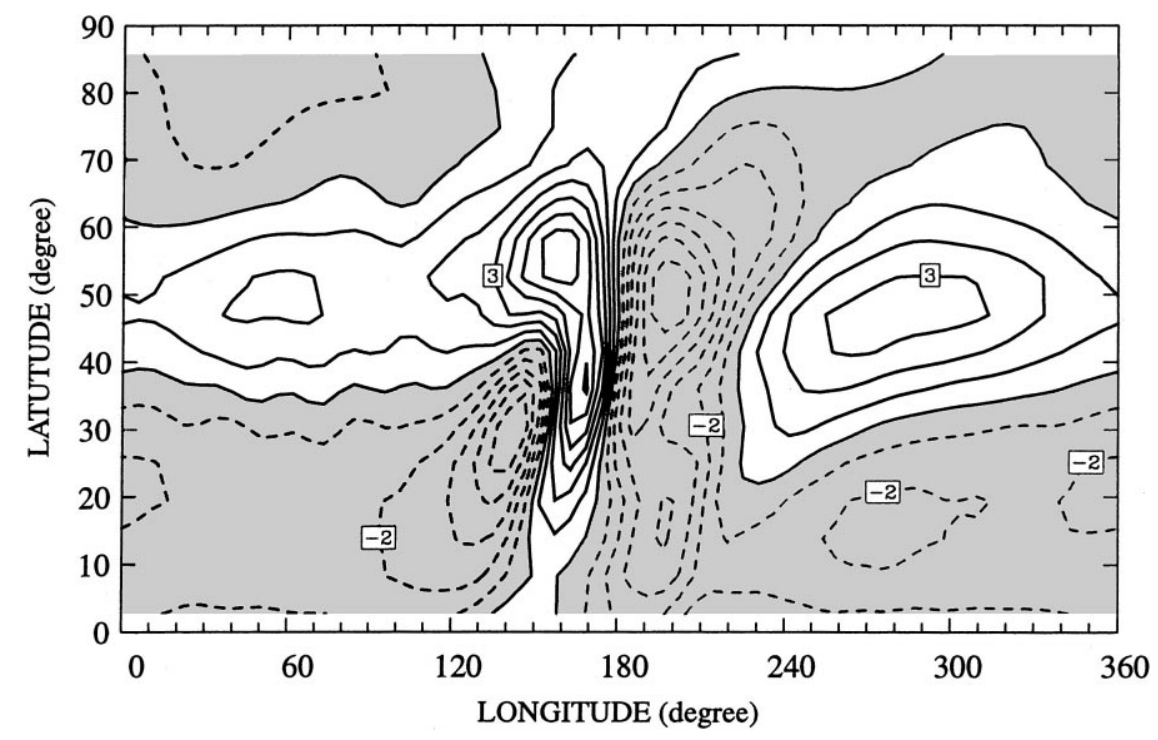

Fig. 10. Meridional winds at the surface of $\sigma=1.0$ from the 3070-day simulation. Contour interval is $1 \mathrm{~m} / \mathrm{s}$, and southerlies are shaded.

the mountain is approximately balanced by poleward thermal advection. Similarly, advection of heat by equatorward winds should occur downstream from the mountain to balance the adiabatic warming. The meridional winds on the lowest sigma surface in Fig. 10 indicate that this simple GCM's response to orographic forcing is consistent with their arguments.

\section{Conclusions}

A full-Galerkin GCM was developed in this study. The linear and nonlinear baroclinicity simulated in this model are examined. The results suggest that this GCM reasonably models the linear baroclinic normal mode and that the finite-element method resolves the vertical structure of the baroclinic normal mode better than the finite-difference method. This advantage is believed to be a result of the higher accuracy of the finite-element method. Reasonable lifecycle behaviors of baroclinic waves are also simulated in this GCM. The structure and flux properties of the nonlinear baroclinic waves are, as expected, closer to the observed baroclinic waves than those predicted by linear theory. The generation, propagation, and decay of baroclinic waves are well simulated in this model. This lifecycle calculation indicates that this GCM is capable of simulating the evolution of nonlinear baroclinic waves.

A long-term integration was carried out with a zonally symmetric forcing in the GCM. The model climate derived from this extended integration was investigated. The time-mean statistics of the zonal mean circulations and transient eddy properties were documented and compared between the no-mountain hemisphere and the mountain hemisphere. Comparisons between climates in the mountain atmosphere and in the no-mountain atmosphere suggest that the presence of mountain does not alter the meridional structure of the zonal mean circulation. Comparisons of these time-mean statistics to observed winter time statistics in the real atmosphere indicate that this GCM produces reasonable general circulations of 
the atmosphere. In conclusion, this full-Galerkin method GCM produces reasonable simulations of atmospheric baroclinic waves and general circulation, and is therefore suitable for simulation of atmospheric climate and variability.

\section{Nomenclature}

\begin{tabular}{|c|c|c|}
\hline$D$ & \multicolumn{2}{|l|}{ divergence } \\
\hline$f$ & \multicolumn{2}{|c|}{ Coriolis parameter } \\
\hline $\boldsymbol{F}=\left(F_{x}, F_{y}\right)$ & \multicolumn{2}{|c|}{ vector of horizontal friction force per unit mass } \\
\hline$H_{T}$ & \multicolumn{2}{|c|}{ adiabatic heating } \\
\hline$q=\ln p_{\mathrm{s}}$ & \multicolumn{2}{|c|}{ logarithm of surface pressure } \\
\hline$t$ & \multicolumn{2}{|l|}{ time } \\
\hline$T$ & \multicolumn{2}{|l|}{ temperature } \\
\hline $\boldsymbol{V}=(u, v)$ & \multicolumn{2}{|c|}{ horizontal wind vector } \\
\hline \multicolumn{3}{|l|}{ Greek letters } \\
\hline$\phi$ & & latitude \\
\hline$\Phi$ & & geopotential height \\
\hline & static stability \\
\hline \multicolumn{2}{|c|}{$\gamma=\left(\Lambda I / C_{\mathrm{p}}\right)-(01 / 00)$} & longitude \\
\hline \multicolumn{2}{|l|}{$\mu$} & $\sin \phi$ \\
\hline \multicolumn{2}{|l|}{$\begin{array}{l}v \\
\dot{\sigma}\end{array}$} & diffusive coefficient for bi-harmonic diffusion \\
\hline \multirow{2}{*}{\multicolumn{2}{|c|}{$\begin{array}{l}\dot{\sigma} \\
\xi\end{array}$}} & vertical motion in sigma coordinates \\
\hline & & relative vorticity \\
\hline
\end{tabular}

\section{Acknowledgements}

This paper is based on the work that the author conducted at the Department of Atmospheric Sciences of the University of Washington. The author thanks Professor Dennis L. Hartmann for his support during this study. This paper was written when the author was supported at University of California, Los Angeles by NOAA GOALS Grant NA66GP0121.

\section{References}

[1] A. Arakawa, Y. Mintz, The UCLA atmospheric general circulation model, Technical Report, Department of Atmospheric Sciences, University of California, Los Angeles, CA, 1974.

[2] R. Asselin, Frequency filter for time integrations, Mon. Wea. Rev. 100 (1972) 487-490.

[3] M. Béland, C. Beaudoin, A global spectral model with a finite-element formulation for the vertical discretization: adiabatic formulation, Mon. Wea. Rev. 113 (1985) 1207-1910.

[4] E.N. Blinova, Dokl. Akad. Nauk SSSR (Rept. Acad. Sci. USSR) 39 (1943) 284.

[5] W. Bourke, A multilevel spectral model. Part I: Formulation and hemispheric integrations, Mon. Wea. Rev. 102 (1974) 687-701.

[6] J.G. Charney, The dynamics of long waves in a baroclinic westerly current, J. Meteorol. 4 (1947) 135-162. 
[7] R. Daley, C. Girard, J. Henderson, I. Simmonds, Short-term forecasting with a multilevel spectral primitive equation model. Part I: Model formulation, Atmosphere 14 (1976) 98-134.

[8] E.T. Eady, Long waves and cyclone waves, Tellus 1 (3) (1949) 33-52.

[9] E.B. Eliasen, B. Machenhauer, E. Rasmussen, On a numerical method for integration of the hydrodynamical equations with a spectral representation of the horizontal fields, Report No. 2, Institute of Theoretical Meteorology, Copenhagan University, Copenhagan, 1970, 35 pp.

[10] B.J. Hoskins, A.J. Simmons, A multi-layer spectral model and the semi-implicit method, Q. J. R. Meteorol. Soc. 101 (1975) 637-655.

[11] B.J. Hoskins, D.J. Karoly, The steady linear response of a spherical atmospheric to thermal and orographic forcing, J. Atmos. Sci. 38 (1981) 1179-1196.

[12] E.N. Lorenz, Available potential energy and the maintenance of the general circulation, Tellus 2 (1955) $157-167$.

[13] S. Manabe, J. Smagorinsky, R.F. Strickler, Simulated climatology of a general circulation model with a hydrologic cycle, Mon. Wea. Rev. 93 (1965) 769-798.

[14] F. Mesinger, A. Arakawa, Numerical Methods used in Atmospheric Models, Vol. I, GARP Publication Series, No. 17, WMO, Geneva, Switzerland, 1976.

[15] A.H. Oort, Global atmospheric circulation statistics 1958-1973, NOAA Professional Paper, Vol. 14, NOAA, 1983,180 pp.

[16] N.A. Philips, A coordinate system having some special advantages for numerical forecasting, J. Meteorol. 14 (1957) 184-185.

[17] A.J. Robert, J. Henderson, C. Turnbull, An implicit time integration scheme for baroclinic models of the atmosphere, Mon. Wea. Rev. 100 (1972) 329-335.

[18] I. Silberman, Planetary waves in the atmosphere, J. Meteorol. 11 (1954) 27-34.

[19] A.J. Simmons, B.J. Hoskins, Baroclinic instability on the sphere: normal modes of the primitive and quasi-geostrophic equations, J. Atmos. Sci. 33 (1976) 1454-1477.

[20] A.J. Simmons, B.J. Hoskins, Baroclinic instability on the sphere: solutions with a more realistic tropopause, J. Atmos. Sci. 34 (1977) 581-588.

[21] A.J. Simmons, B.J. Hoskins, The life-cycles of some nonlinear baroclinic waves, J. Atmos. Sci. 35 (1978) $414-432$.

[22] A.N. Staniforth, R.W. Daley, A finite-element formulation for the vertical discretization of sigma-coordinate primitive-equation models, Mon. Wea. Rev. 105 (1977) 1108-1118.

[23] J.M. Wallace, The climatological mean stationary waves: observational evidence, in: B. Hoskins, R. Pearce (Ed.), Large-scale Dynamical Processes in the Atmosphere, Academic Press, 1983, 397 pp.

[24] J.-Y. Yu, The role of wave-mean flow interaction and orographic forcing on the generation of low-frequency variability in a newly developed GCM, Ph.D. Dissertation, University of Washington, Seattle, Washington, 1993, 236 pp. 